\title{
Insights into Collaborative Platforms for Social Media Use Cases
}

\author{
George SUCIU ${ }^{1 *}$, Corentin BOSCHER ${ }^{1}$, Laura PRIOUX ${ }^{1}$, Adrian PASAT ${ }^{1}$, Ciprian DOBRE ${ }^{2}$ \\ ${ }^{1}$ Beia Research Department, 16-22 Peroni, Bucharest, 041386, Romania \\ george@beia.ro (*Corresponding author); \{corentin.boscher, laura.prioux, adrian.pasat\}@beia.ro \\ ${ }^{2}$ National Institute for Research \& Development in Informatics, 8-10 Maresal Al. Averescu Avenue, sector 1, \\ Bucharest, 011455, Romania \\ ciprian.dobre@ici.ro
}

\begin{abstract}
The first social network has been created 30 years ago and allowed users to create a profile, add friends or use chat rooms. In time social media evolved to social platforms like Facebook, Twitter or Instagram, which in fact are a huge mine of information for brands, vendors or even companies. Thus, marketing and recruiting processes are parts of today's social media. By analyzing and understanding consumers' activity on social media, companies have a powerful tool available for use. They can develop their advertising campaign or as close as possible to the needs of users or target most relevant applicants' profiles for recruiting purposes. In this context, we bring an innovative platform that integrates Digital Interaction Intelligence (DII) for data processing based on intelligent techniques and Digital Interaction Data (DID) for data aggregation, persistence and user interaction. Building such a platform required to study, compare and select the adequate collaborative platform for our goals, having in mind a scalable design for further developments in other use cases.
\end{abstract}

Keywords: Social media, Recruiting, Marketing, Digital Interaction.

\section{Introduction}

Every day, new users create accounts on a large number of currently available social media. Sharing pictures, videos, interact with friends, comment publication or like content are common actions on this type of applications. All these actions became really useful in the professional work worldwide. In fact, they can be relevant to improve ad campaign impact or recruiting methods. Now, the problem is how to collect all the user's interactions, activity or profile information on social networks, sort and process them to get the more relevant, accurate and useful information. Several specialized companies already provide tools that use analysis methods like sentiment analysis, and searching engine to sort users profile for example. The objective is to build an architecture based on a Big Data platform that enables the process of incoming data. The whole architecture is composed of two main modules: DID (Digital Interaction Data) and DII (Digital Interaction Intelligence). The paper is organized as follows. In section 2 we give examples of tools and present two general methods used by them. After that, we explain in section 3 the proposed use cases: recruiting and marketing. Then, in section 4 we compare collaborative tools in which we aim to integrate use cases functions and finally, we draw the conclusions in section 5 .

\section{Related work}

Similar project/platform for social media cloud call center will be presented in this section. These projects are oriented towards social networks used for marketing and recruiting operations. Moreover, we will present two methods used in relation to this project: text mining and sentiment analysis. Text mining is a technique for automating the processing of large volumes of textual content. Text mining can be used in the context of marketing studies, monitoring or social intelligence. Sentiment analysis which is also called opinion mining corresponds to build a system that collects and categorize opinions about a product or an ad campaign [9]. Automated sentiment analysis often uses machine learning, which is a type of artificial intelligence, to mine text for sentiment. This paper [6] provides insight on how to use text data processing techniques and analysis for effective managerial decisions. Performing quantitative and qualitative surveys of customer satisfaction using information technology in the form of computer-aided prove to be very efficient for solving real problems of quality management.

\subsection{SmashFly}

SmashFly [4], founded in 2007 by Mike Hennessy, developed a new category of human resources technology. SmashFly is a platform dedicated to recruitment marketing expertise so it allows companies to improve their recruitment processes. SmashFly's Enterprise Recruitment Marketing Platform (RMP) works like an engine 
that continuously markets brand, content, and jobs of the enterprise to boost awareness and nurture leads, converting them into quality applicants.

The Customer Relationship Management (CRM) of the platform proactively creates a network of potential candidates and makes it easier for them to find a job. The platform also makes it easier for candidates to change their careers. SmashFly offers users a simple way to create their own dashboards. They can insert all the information essential to their marketing, in real time, to have statistics and to obtain the best return on investment.

\subsection{LinkedIn Talent Solutions}

LinkedIn Talent Solutions [1] gives access to LinkedIn's massive member network: more than 500 million people. Thanks to this tool, recruiters can find the right candidates with the search tools, guided search, advanced recruiting filters (more than 20) or the search based on the ideal candidate for the company. To contact the candidate, recruiters can personalize InMail for one candidate or a group of candidates with a limit of 150 messages per month. Talent solution can provide custom job recommendations based on the user profile thanks to an automatic recommendation of company job offers to LinkedIn members who have the skills and expertise needed. Companies have the access to several management tools that review and filter candidates, save their status and contact them with InMail.

\subsection{Zoomph}

Zoomph [5] envisions to collect, classify \& activate information instantaneously to amplify digital marketing \& understanding. More precisely, Zoomph provides social recruiting and social commerce solutions.

Social recruiting allows companies to create their own strategy for acquiring passive and active job candidates using Zoomph's social listening tools. Zoomph's tools capture social signals identifying a person's qualifications, passions, and requirements for a job. It is also possible to enable notifications when a candidate posts something especially influential or relevant to the company [5].

The platform also allows interacting with candidates by publishing content that teases their interests. Social commerce provides tools to manage and build commerce campaign. First, the tool helps companies to know their audience or find opportunities to expand. Afterwards, Zoomph provides a craft tool to build an online experience with social hubs, microsites or contests framework. Social commerce solution also provides tools to promote hub company's and moderate publish content. Companies can finally track, compare and optimize their campaign with a dashboard.

\subsection{Retargeting}

Retargeting [8] is an advertising practice that most often involves targeting an individual who has visited a website but for which there was no purchase or transformation during the visit. It is possible to have a vision of the activity of each visitor. An algorithm has been set up to follow each visitor, thus performing a profiling process. Personalized messages are sent automatically to each visitor based on their actions.

The sending can be done by SMS, e-mail or directly on the website. A dashboard allows the efficient analysis of the actions of the visitors. Statistics about sending SMS, e-mail or message via the website are also visible, a full analysis of the results can then be done by the user of the platform. Based on these results, the marketing strategy can be modified.

\subsection{Sentiment analysis tools for Twitter}

Several experiments have already been carried out regarding the analysis of feelings on social networks like Twitter. In fact, The Sri-Lanka Faculty of Information Technology created a platform with four main modules naming, crawler, sentiment analysis tool, data mining module and dashboard [7].

Firstly, the crawler gathers social media data to a local data source for ease of analysis. For this, it had to use a Twitter API to get access to Twitter data. The main part of the platform is the sentiment analysis tool which is composed of: extraction methods, a classifier, and a word sense disambiguation. The last important feature is the data mining part that allows product profiling, trend analysis, and forecasting works. Finally, the result of data mining and sentiment analysis is displayed on a dashboard.

\subsection{Sentiment analysis tools for Facebook}

A sentiment analysis study was performed on more over than 1000 Facebook posts about 
newscasts, comparing the sentiment for Rai - the Italian public broadcasting service - towards the emerging and more dynamic private company.

The sentiment and knowledge mining system used in this paper [3] is built on the following components:

- a crawler (retrieves data);

- a semantic engine (identification of knowledge);

- a machine translation engine (automatic translation), geo-referencing engine (interactive geographical representation), classification engine (classification of search results into clusters). Authors conclude their experiment by explaining that monitoring the social media activities is an efficient way to measure customers' interests and keep track of their sentiment towards certain brands or products.

\subsection{Text mining for retail stores}

A text mining study was conducted to extract information about real-time events from social networks. Eman M.G. Younis presents in his article [2] an open-source approach that uses text mining through posts on Twitter. He explains how he contributed to the marketing of two English retail stores, thanks to text mining and social media. Consumer satisfaction surveys are expensive for companies and can take a long time.

To know the opinions of consumers, the approach used by the author is to retrieve the tweets on the stores and analyze whether these tweets are positive or negative for the store. Once the results have been provided to the stores, they can quickly get an idea of their marketing strategy and know what consumers think of their store. The authors conclude their paper by explaining that this kind of works will provide a competitive advantage for business retailers and service providers. This will help them analyze, via social networks, the opinions of their customers regarding their product or service. Thus, they can improve their business value and better manage their customer relationships.

\subsection{Text mining for natural disaster}

The aim of the study [10] is to monitor the flow of text from social media to monitor possible natural disasters. During a natural disaster, the easiest way to communicate this information is to publish it via a microblogging. The application then makes it possible to quickly detect the event by extracting the data from this microblogging. The authors use Twitter to run their experiment, they consider every Twitter user as a sensor to monitor recent tweets and thus detect natural disasters.

To detect events, the application works like this:

- Classification of events. To do this, the classifier is trained by using keywords, message length, and the corresponding context as features to classify tweets into positive or negative cases.

- Find the location of the event thanks to a spatiotemporal model build to target event and then identify the location

- This work has already been validated by Japanese who have opted for this solution because every year there are many earthquakes as well as many microblogging users to post the necessary information.

\subsection{Social media for internship}

In India, students learn how to use social media to their advantage and how to attract or find employers using social media platform such as LinkedIn [4]. At the beginning of the courses, students were asked to sum up their skills. Then, they were asked to make corrections and improvements on them. They finally produced a resume of their professional capabilities. Thanks to their new complete and efficient social media profile, students will be able to apply to internship missions. This type of event has been created because, in India, a large percentage of graduated engineers are not ready to integrate themselves into professional life after they have completed their studies. The main reason is that the colleges teach outdated courses to students. This caused a large gap between the curriculum and the industry requirements. This problem results in graduates who are unemployable without training in basic skills of the industry, which is tackled also by the SoMeDi [11] project. That's why social media is important for students to find an internship that will help them to learn many basics knowledge about corporate industries.

\section{Social media use cases}

The main objective is to enable end users (companies) to improve their social business intelligence: giving a tailored recommendation, development of new product or services from harvesting ideas, improving customers' retention 


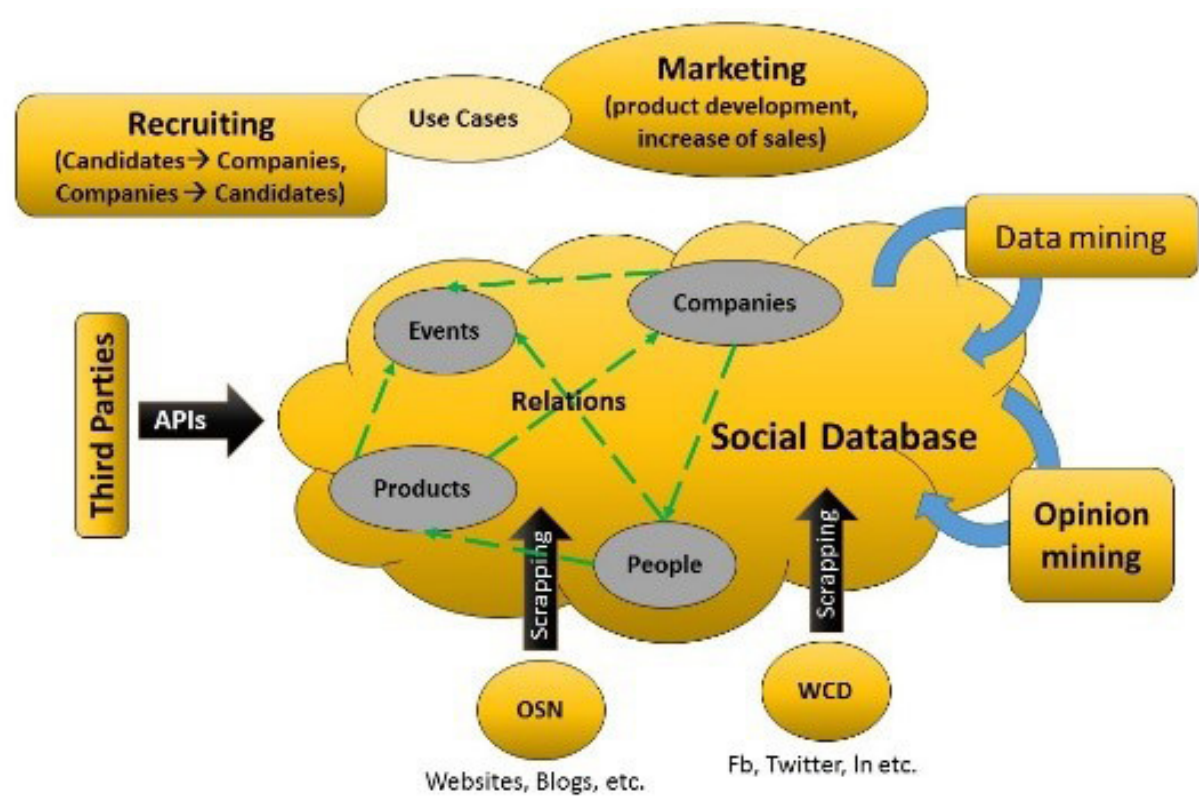

Figure 1. Platform diagram architecture

for products, content and/or services. This objective will be reflected in the application use cases envisaged in [11]. Figure 1 represents the platform diagram architecture.

\subsection{Marketing Use Case}

The main objective of this use case is to improve the marketing performance of companies in two different sectors: e-commerce and restaurants with social media presence including promotions and marketing campaigns. By means of DII tools provided, forums (OSN scrapping module) and social networks (WCD scrapping module) monitoring in order to check the impact of their marketing campaigns will be implemented to generate recommendations and automatized changes which can be used to increase sales volume and business impact.

\subsection{Recruiting Use Case}

Tools developed will be used for mining data from professional networks, opinion mining on social media platforms to provide a personalized recommendation, evaluation of internship and/or apprenticeship programs offered by companies. The platform will use data collected from social media sources, and data uploaded into the platform.

\section{The comparative analysis of collaborative platforms}

A collaboration platform is a category of business software that adds lots of social networking capabilities to work processes. In fact, collaborative platforms are tools for bringing together all the members of a company, an association, a school, etc. and give them access to all the tools and information they need [12]. They can create groups for projects to work as a team, a customizable messaging or chat service, integrate external applications to manage documents, calendars, tasks, etc. All these features available with today's collaborative platforms will significantly improve productivity, internal communication and time-saving.

Also, and most importantly through these tools we will shift towards a new paradigm collaborative media. Through the advanced algorithms that analyze the metadata of digital interactions we can alter the communication channels so that consumer will not be a passive recipient, but the originator or even collaborator.

However, currently available platforms don't provide the same services, level of customization or support for users for example. The goal is to analyze the tools available that allow a high degree of modularity, as is desired to have the possibility to integrate different modules personalized to each use case, so we analyzed several collaborative platforms and studied their modularity capabilities, as presented in Table 1 .

These modules are responsible for collecting data from the internet (i.e., DW website and social media). It acts as an interface of the platform to interact with the rest of the world. The collected 
Table 1. Collaboration tools comparison

\begin{tabular}{|c|c|c|c|c|c|}
\hline Tool name & Platforms & Communications & Security & $\begin{array}{l}\text { Cloud services } \\
\text { and storage }\end{array}$ & $\begin{array}{l}\text { API/SDK } \\
\text { (frameworks) }\end{array}$ \\
\hline Slack & $\begin{array}{l}\text { Mobile: iOS, } \\
\text { AndroidDesktop: } \\
\text { Mac, Windows, } \\
\text { Linux Browser : } \\
\text { Google Chrome } \\
\text { recommended }\end{array}$ & $\begin{array}{l}\text { Screen sharing, } \\
\text { simple/group } \\
\text { voice and video } \\
\text { call, file Sharing }\end{array}$ & $\begin{array}{l}\text { Data encryption } \\
\text { in transit and } \\
\text { at rest; Custom } \\
\text { message } \\
\text { retention; OAuth } \\
\text { via Google }\end{array}$ & $\begin{array}{l}\text { Google Drive, } \\
\text { Dropbox; } 5 \mathrm{~GB} \text { up } \\
\text { to } 20 \mathrm{~GB}\end{array}$ & $\begin{array}{l}\text { SDK: Python, } \\
\text { Node.js, Hubot; } \\
\text { API: C\#, Go, } \\
\text { JavaScript, PHP, } \\
\text { Python, Ruby; }\end{array}$ \\
\hline Bitrix24 & $\begin{array}{l}\text { Mobile: iOS, } \\
\text { Android Desktop: } \\
\text { Mac, Windows, } \\
\text { Linux }\end{array}$ & $\begin{array}{l}\text { Tasks and } \\
\text { projects, chat and } \\
\text { video, calendars, } \\
\text { mail, document } \\
\text { sharing }\end{array}$ & $\begin{array}{l}\text { SSL-encrypted } \\
\text { connection } \\
\text { (with a 256-bit } \\
\text { key), two factor } \\
\text { authorization, } \\
\text { RSA encryption }\end{array}$ & $\begin{array}{l}\text { Bitrix 24.drive; } \\
\text { 5GB up to } \\
\text { unlimited }\end{array}$ & $\begin{array}{l}\text { API: JavaScript, } \\
\text { HTML, PHP }\end{array}$ \\
\hline HipChat & $\begin{array}{l}\text { Mobile: iOS, } \\
\text { Android Desktop: } \\
\text { Mac, Windows, } \\
\text { Linux }\end{array}$ & $\begin{array}{l}\text { Group chat, } \\
\text { video chat, screen } \\
\text { sharing, file } \\
\text { storage }\end{array}$ & $\begin{array}{l}\text { 256-bit SSL } \\
\text { encryption; } \\
\text { control guest } \\
\text { access }\end{array}$ & $\begin{array}{l}\text { Dropbox, Google } \\
\text { Drive, Zapier; } \\
\text { 5GB up to } \\
\text { unlimited }\end{array}$ & $\begin{array}{l}\text { SDK: Node. } \\
\text { js, PythonAPI: } \\
\text { GO, Java, Node. } \\
\text { js, Perl, PHP, } \\
\text { Python, Ruby, } \\
\text {.NET, Scala }\end{array}$ \\
\hline Flowdock & $\begin{array}{l}\text { Mobile: iOS, } \\
\text { Android Desktop: } \\
\text { Mac, Windows, } \\
\text { Linux }\end{array}$ & $\begin{array}{l}\text { instant video chat, } \\
\text { screen sharing, } \\
\text { file sharing }\end{array}$ & $\begin{array}{l}\text { Public key } \\
\text { to encrypt } \\
\text { any sensitive } \\
\text { information, } \\
\text { encrypted } \\
\text { password storage }\end{array}$ & Zapier & $\begin{array}{l}\text { API: Ruby, R, } \\
\text { Python, PHP, } \\
\text { Perl, JavaScript, } \\
\text { Haskell, Go, } \\
\text { Erlang, Clojure, }\end{array}$ \\
\hline WIMI & $\begin{array}{l}\text { Mobile: iOS, } \\
\text { Android Desktop: } \\
\text { Mac, Windows }\end{array}$ & $\begin{array}{l}\text { Screen sharing, } \\
\text { file sharing, } \\
\text { video calls, direct } \\
\text { messages }\end{array}$ & $\begin{array}{l}\text { SSL 256-bits } \\
\text { AES, asymmetric } \\
\text { encryption, }\end{array}$ & $\begin{array}{l}\text { Wimi drive, } \\
\text { Dropbox, Google } \\
\text { drive, Box, } \\
\text { OneDrive; } 10 \mathrm{~GB} \\
\text { up to } 500 \mathrm{~GB}\end{array}$ & $\begin{array}{l}\text { Office } 365, \\
\text { Google Calendar, } \\
\text { Evernote, } \\
\text { FreshBooks, } \\
\text { Skype, Gmail, } \\
\text { Slack }\end{array}$ \\
\hline
\end{tabular}

data is stored in the platform for subsequent analysis. Specifically, ingestion modules must be capable of storing the collected data in the HDFS (Hadoop Distributed File System) of the platform. Some of the ingestion modules in the platform are described below:

- The Twitter crawler collects tweets according to the selected keywords. This component is based on Python and can be deployed as a web service. As such, and because the limitation for retrieving tweets is on the quotas of the Twitter API and not in the processing capabilities, this element is to be deployed as a single instance.

- The Facebook media crawler will collect public Facebook posts according to the selected projects keywords. This crawler will run as an application and will be deployed in a single instance, because the Facebook API is also limited with quotas per application.

- YouTube is a social video-sharing network. Each video can be commented by YouTube users. YouTube offers an API to access to the information needed: recollect the comments from a specific video. The YouTube Crawler has been developed using Python and Google API Python Client library.

\section{Conclusions}

In this paper, we presented an analysis of one of the most important components within social media media use cases, namely selection of collaborative tools. The main goal is to unlock the value hidden in the digital content and to trace human interactions through the application of advanced artificial intelligence and machine learning techniques. Hence, after documenting and presenting some of the technologies used in other applications, like sentiment analysis and text mining use cases, we directed our focus on several collaborative platforms and analyzed the possibilities to integrate some of the modules in the proposed DII architecture.

\section{Acknowledgements}

This work was supported by a grant of the Ministry of Innovation and Research, POC-5C project. 


\section{REFERENCES}

1. Bali, M. \& Dixit, S. (2016). Employer Brand Building for Effective Talent Management, International Journal of Applied Sciences and Management, 2(1), 183-191.

2. Eman, M. \& Younis, G. (2015). Sentiment Analysis and Text Mining for Social Media Microblogs using Open Source Tools: An Empirical Study, International Journal of Computer Application, 112(5), 975 - 979.

3. Frederico, N., Aliprandi, C., Capeci, F. Cuadro, M. \& By, T. (2012). Sentiment Analysis on Social Media. In IEEE/ACM International Conference on Advances in Social Networks Analysis and Mining (pp. 919-926).

4. Hameed, S. \& Nileena, G. S. (2014). IEEE student quality improvement program: to improve the employability rate of students. In 2014 IEEE International Conference on MOOC, Innovation and Technology in Education (MITE) (pp. 219-222).

5. Hettche, M., Tupper, C. \& Rooney, C. (2017). Using a Social Machine for Promotional Marketing on Campus: A Case Study.

6. Kovács, G., Bogdanova, D., Yussupova, N. \& Boyko, M. (2015). Informatics Tools, AI Models and Methods Used for Automatic Analysis of Customer Satisfaction, Studies in Informatics and Control, 24(3), 261-270. ISSN 1220-1766.
7. Kowcika, A., Gupta, A., Sondhi, K., Shivhre, N. \& Kumar, R. (2013). Sentiment analysis for social media, International Journal of Advanced Research in Computer Science and Software Engineering, 3(7), 216-221.

8. Li, M., Chen, G., Zhang, Z. \& Fu, Y. (2012). A social collaboration platform for enterprise social networking. In 2012 IEEE $16^{\text {th }}$ International Conference on Computer Supported Cooperative Work in Design (CSCWD) (pp. 671-677).

9. Ringsquandl, M. \& Petković, D. (2013). Analyzing Political Sentiment on Twitter, In AAAI Spring Symposium: Analyzing Microtext (pp. 40-47).

10. Sakaki, T., Okazaki, M. \& Matsuo, Y. (2013). Tweet analysis for real-time event detection and earthquake reporting system development, IEEE Transactions on Knowledge and Data Engineering, 25(4), 919-931.

11. Suciu, G., Anwar, M. \& Conu, R. (2017). Social Media and Digital Interactions using Cloud Services for orienting Young People in their Careers. In The International Scientific Conference on eLearning and Software for Education (pp. 419-423).

12. Venkatesh, A. (2016). Collaborative media: production, consumption, and design interventions, Consumption Markets \& Culture Journal, 19(6), 597-600. 